\title{
BIOLOGÍA
}

\section{Quirópteros del campus de la Universidad Nacional de Asunción-Paraguay}

\author{
Katia Airaldi Wood ${ }^{1}$, María Elena Torres ${ }^{1}$, María Belén Barreto¹, \\ Andrea Weiler ${ }^{1}$, Gloria González de Weston", \\ Griselda Zárate ${ }^{1}$, Richards Stevens ${ }^{1}$
}

\section{Resumen}

Introducción: Los murciélagos realizan funciones ecológicas indispensables para el mantenimiento de los ecosistemas, dispersan semillas de varias especies vegetales y contribuyen a la diversidad de dichas especies. Una característica importante de los murciélagos es su capacidad de actuar como indicadores de niveles de perturbación de hábitat por lo que ofrecen una amplia visión de la salud de un ecosistema debido a los diferentes recursos tróficos que explotan. La fauna en zonas urbanizadas, muchas veces es desconocida y se encuentra sumergida en una miscelánea de paisajes donde existen especies poco apreciadas como los murciélagos. El Campus Universitario de la Universidad Nacional de Asunción (UNA), comprende 300 ha, se localiza en San Lorenzo (Dpto. Central, Paraguay). Para el presente estudio, se seleccionaron cuatros sitios, denominados "Mangal", "Piscicultura", "Campo Experimental", y el ultimo sitio diferenciado en "Eucaliptal" y "Bosque Secundario".

Material y Método: Se realizó una salida preliminar (febrero del 2016), y seis salidas de campo de cuatro días cada una (un día por sitio seleccionado) durante agosto, septiembre, octubre, noviembre y diciembre del 2016. En cada sitio se colocaron dos redes de niebla de $8 \mathrm{~m}$ y $6 \mathrm{~m}$ de ancho $x 2,5 \mathrm{~m}$ de alto cada una. Cada red se colocó en simultáneo a la altura del sotobosque a partir de las 16:00-17:00 hs, hasta las 22:00-23:00 hs. Los individuos capturados, fueron almacenados temporalmente en bolsas de tela, y sus datos de colecta fueron registrados en planillas. Los individuos de fácil identificación fueron liberados. Los capturados fueron fotografiados, pesados y medidos: longitud total (LT), longitud cola (LC), longitud pata (LP), longitud oreja (LO) y trago (T). Se prepararon como piel y colección líquida (alcohol al 70\%) y se depositaron en la Colección Zoológica de la FACEN (CZCEN) (Permiso de Colecta de la Secretaría del Ambiente N¹91/2016). También se efectuó la caracterización de la vegetación mediante metodologías establecidas en transectos.

\footnotetext{
1. Departamento de Biología. Colección Zoológica -CZCEN. Facultad de Ciencias Exactas y Naturales -FACEN-, Universidad Nacional de Asunción, Paraguay.

El proyecto fue financiado dentro del marco del Programa PROCIENCIA- CONACYT.

Este trabajo fue presentado en las XXIX Jornadas Argentinas de Mastozoología (2016), actualmente se encuentra en Revisión para ser publicado en el Boletín del Museo Nacional de Historia Natural del Paraguay (BMNHNP).

E-mail: kairaldi@gmail.com

DOI: 10.26885/rcei.foro.2017.15
} 
Resultados: El esfuerzo de muestreo fue de $48.202 \mathrm{~m} 2 \mathrm{~h}$, se capturaron 21 ejemplares de los cuales 17 fueron colectados. Se presenta el primer listado de murciélagos del Campus de la UNA, con 10 especies registradas, incluidas en 6 géneros y 3 familias (Phyllostomidae, Vespertilionidae, Molossidae). Se aportan seis nuevos registros para la localidad de San Lorenzo de las especies Artibeus fimbriatus (Gray, 1838), Artibeus planirostris (Spix, 1823), Artibeus lituratus (Olfers, 1818), Platyrrhinus lineatus (E. Geoffroy, 1810), Myotis nigricans (Schinz, 1821) y Molossops temminckii (Burmeister, 1854).

La riqueza de murciélagos del Campus Universitario de la UNA, representa el 77\% de las especies citadas para San Lorenzo, el 38, 4\% de las citadas para el Dpto. Central, y el 17, $5 \%$ de las especies citadas para el Paraguay. La familia Phyllostomidae fue la más diversa colectada en el Campus, con cuatro especies identificadas y el $47,4 \%$ de las capturas realizadas. La especie $A$. fimbriatus fue una de las especies con mayor número de captura (19\%), y A. lituratus fue la especie menos registrada durante el estudio $(0,4 \%)$, ambas registradas únicamente en el Mangal. Otras especies registradas con frecuencia con el $14 \%$ de captura, fueron A. planirostris, asociado al Mangal y al Bosque Secundario y $P$. lineatus capturado en el Mangal y el Campo Experimental.

En cuanto a los murciélagos insectívoros registrados en el área de estudio, los pertenecientes a la Familia Vespertilionidae fueron las siguientes especies con mayor número de captura (19\%): Eptesicus furinalis (d Orbigny, 1847) (Piscicultura y Campo Experimental) y $M$. nigricans (Bosque Secundario). Los insectívoros verspertiliónidos registrados $E$. furinalis y $M$. nigricans, son especies comunes de amplia distribución nacional.

La familia Molossidae, también del gremio de los insectívoros, fue la menos frecuente (9\%) con una sola especie colectada con red de niebla: M. temminckii. Durante el estudio la especie Molossus rufus (E. Geoffroy, 1805) fue capturada de forma ocasional, asociada a edificaciones.

Los sitios que presentaron mayor diversidad de especies en el Campus de la UNA fueron: el Mangal con cuatro especies frugívoras de la familia Phyllostomidae (A. fimbriatus, A. planirostris, A. lituratus, P. lineatus) y el $42,8 \%$ de las capturas realizadas, y el Bosque Secundario con dos familias y dos especies (Phyllostomidae: A. planirostris y Vespertilionidae: M. nigricans) y el $23,8 \%$ de las capturas. En el sitio Piscicultura se registró sólo una especie de la familia Vespertilionidae: E. furinalis.

Conclusiones: El Campus de la UNA alberga aproximadamente el $20 \%$ de la quiropterofauna del Paraguay, por lo cual se sugiere como sitio de importancia para la conservación de los mismos. Se recomienda la incorporación de otros métodos de muestreo, a fin de aumentar el conocimiento de la diversidad local.

Palabras clave: Murciélagos, zonas antrópica, San Lorenzo, Departamento Central. 


\section{Referencias}

Ballesteros, C.J. \& J. Racero-Casarrubia. (2012). Murciélagos del área urbana en la ciudad de Montería, Córdoba - Colombia. Rev. MVZ Córdoba, 17(3), 3193-3199.

Fenton M.B, L. Acharya, D. Audet, M. B. C. Hickey, C. Merriman, M.K. Obrist, D.M. Syme \& B. Adkins. (1992). Phyllostomid bats (Chiroptera: Phyllostomidae) as indicators of habitat disruption in the Neotropics. Biotropica, (24), 440-446.

González, J. G. (1998). Dispersión de semillas por murciélagos: su importancia en la conservación y regeneración del bosque tropical. Acta Zoológica Mexicana (73): 57-74.

Keel, S., Sayre, R. \& Sedaghatkish, G. (2002). Estudios de vegetación de plantas (70-89). In: R. Sayre, Roca E., G. Sedaghatkish \& B. Young. Un Enfoque en la Naturaleza: Evaluaciones Ecológicas Rápidas. The Nature Conservancy. USA.

López-González, C. (2005). Murciélagos del Paraguay. Comité Español del Programa MaB y de la Red IberoMaB de la UNESCO. 